\title{
Studying the Effects of Reaction, Diffusion and Reaeration on River Pollution Using Numerical Simulation of Diffusion- Reaction Equations
}

\author{
Tsegaye Simon, Purnachandra Rao Koya* \\ School of Mathematical and Statistical Sciences, Hawassa University, Hawassa, Ethiopia
}

\begin{abstract}
The present paper addresses diffusion-reaction equation describing the dynamics of both dissolved oxygen and biochemical oxygen demand in case of river pollution. Numerical solutions are obtained and some important inferences are drawn through simulation study. The diffusion - reaction equation is characterized by the reaction term whenever it depends on concentration of the contaminants and in this case the original single diffusion - reaction equation will evolve to be a system of equations. This system of equations develops problem in finding analytical solution. In order to tackle this kind of problem one needs to consider numerical approximation. Hence, we employed numerical methods here in. For that purpose we first separated diffusion term from non-linear reaction term using splitting method. Then we applied numerical techniques such as Galerkin finite element method with linear basis function and improved Runge-Kutta method of order six respectively. To explicitly show the efficacy of these numerical methods, the interaction of dissolved oxygen and biochemical oxygen demand is simulated pertaining to the unsteady flow situations. Furthermore, the impact of parameters such as diffusion and reaeration coefficients has been simulated and observed the dynamics of DO and BOD. It is noticed that the higher are diffusion and reaeration coefficients, the faster is the river purity.
\end{abstract}

Keywords: River pollution, Dissolved oxygen, Biological oxygen demand, Diffusion-Reaction equation, Splitting method, Finite element method, Improved Runge-Kutta method of order six

\section{Introduction}

Agricultural, industrial and domestic activities are significant contributors to the contamination of water bodies e.g., lakes, rivers, oceans, aquifers and groundwater. This contaminated water affects negatively the survival of tiny creatures and also human health. Every year, approximately 25 million people die as a result of water pollution in the world $[5,15]$.

Mathematical models have been used broadly to predict water quality, and to provide reliable tools for its quality management in affected areas. These models are used to simulate the spatial and temporal distributions of various variables related to water quality in the physical areas of study. The use of mathematical models for the simulations is to enhance the decision supporting tools for water resource management. There are many factors of water quality that are required to be observed and used in the models such as dissolved oxygen level, water velocity, pollutant addition, and saturated oxygen concentration. The main consideration here is aimed to predict the amounts of dissolved oxygen $(D O)$ and biochemical oxygen demand $(B O D)$ along a river channel as well as the interaction between these two factors.

Recently, a number of mathematical models on river pollution have been appeared in literature $[1,5,8$, $11-13,15,19-20]$. Historically, the famous mathematical model predicting water quality in a river is proposed by Streeter H. W. and Phelps E. B. [22]. Their model is applied to model the amount of $D O$ in a stream provided that the waste water is discharged into the stream. They assumed that deoxygenation was a first order chemical reaction. Thus, their model was simpler for having an analytical solution. However, for some extended and complicated models the analytical solutions cannot be easily derived. It requires an efficient numerical method to find approximate solution instead. Previously, various numerical methods have been employed in solving pollution model $[1,5,19-20]$.

In this study, we present a mathematical model for $D O$ concentration interaction with BOD during river pollution. The present model equations are in the similar form as those presented in [19]. Additionally, we have proposed and considered different fast, reliable and accurate numerical methods and investigated the reaeration effects on river pollution. The numerical scheme applied here is based on the splitting method, Galerkin finite element method with linear basis function and improved Runge-Kutta method of order six. Furthermore, the effects of diffusion and reaction are also investigated by including these terms in the model, and it is then solved numerically. 


\section{Mathematical Model}

Once the pollution is injected into the river, it is then convected while diffusing through turbulent mixing. Simultaneously, it chemically reacts with the $D O$ causing deoxygenation in the river that is counteracted by the transport of oxygen from the atmosphere, known as reaeration, across the air-water interface acting as a permeable membrane [1, 19 - 20]. This process, in case of a moderately polluted river, can be described mathematically as

$$
\begin{aligned}
& \frac{\partial u_{1}}{\partial t}=-w \frac{\partial u_{1}}{\partial x}+D_{1} \frac{\partial^{2} u_{1}}{\partial x^{2}}+\lambda\left(\omega-u_{1}\right)-\gamma u_{1} u_{2} \\
& \frac{\partial u_{2}}{\partial t}=-w \frac{\partial u_{2}}{\partial x}+D_{2} \frac{\partial^{2} u_{2}}{\partial x^{2}}-\gamma u_{1} u_{2}
\end{aligned}
$$

The notations and description of variables and parameters are listed in Table 1.

For simplicity, let us assume that advection along the river is neglected or ignored, that is $w=0$. This assumption reduces the well-known computational difficulties that are associated with advection dominated flows. Also it helps to resolve the problem in an economical fashion; the transport, diffusion and interaction of $D O$ and $B O D$ for hundreds of kilometers. Upon using this assumption, we obtain the Lagrangian form of equations (1) and (2) as

$$
\begin{aligned}
& \frac{\partial u_{1}}{\partial t}=D_{1} \frac{\partial^{2} u_{1}}{\partial x^{2}}+\lambda\left(\omega-u_{1}\right)-\gamma u_{1} u_{2}, \quad \text { (Oxygen) }(3) \\
& \frac{\partial u_{2}}{\partial t}=D_{2} \frac{\partial^{2} u_{2}}{\partial x^{2}}-\gamma u_{1} u_{2} . \quad(\text { BOD })(4)
\end{aligned}
$$

\begin{tabular}{|c|l|}
\hline VariablesandParameters & Description \\
\hline$x$ & Distance along the river \\
\hline$t$ & Time \\
\hline$u_{1}$ & $D O$ concentration \\
\hline$u_{2}$ & $B O D$ concentration \\
\hline$w$ & River velocity \\
\hline$D_{1}$ & $D O$ diffusion coefficient \\
\hline$D_{2}$ & $B O D$ diffusion coefficient \\
\hline$\lambda$ & Reaeration rate \\
\hline$\omega$ & $\begin{array}{l}\text { Concentration of oxygen in the air immediately } \\
\text { above the river }\end{array}$ \\
\hline$\gamma$ & Reaction rate of $D O$ and $B O D$ \\
\hline
\end{tabular}

Table 1 Notational Glossary of Variables and Parameters

In order to simplify the equations (3) and (4), we will assign constant values for all the parameters $\gamma, \omega$, $\lambda, D_{1}$ and $D_{2}$ and then carry out simulation study.

\section{Numerical Approximation}

We now describe some numerical approximation techniques those will be applied on equations (3) and (4) together with appropriate initial and boundary conditions. Here it is appropriate to introduce, separately, the details of each of the three principal features of the methods, viz., splitting, finite element, and improved RungeKutta of order six schemes.

\subsection{Splitting Method}

In many applications mixing of various terms in the equations for the discretization and solver methods make it difficult to solve them together. Hence, we use the splitting method to decouple a complex equation into various simpler equations and to solve them with adapted discretization and solver methods. The adapted methods for a simpler equation give improved results for simpler parts. The operator splitting is an attractive technique for solving coupled systems of partial differential equations of the type (3) and (4). Since complex system of equations are split into simpler parts those are easier to solve. Here we now separate the non-linear reaction term from diffusion term so that

$$
\begin{aligned}
& \frac{\partial u_{1}}{\partial t}= D_{1} \frac{\partial^{2} u_{1}}{\partial x^{2}} \\
& \frac{\partial u_{2}}{\partial t}=D_{2} \frac{\partial^{2} u_{2}}{\partial x^{2}} \\
& \frac{\partial u_{1}}{\partial t}=\lambda\left(\omega-u_{1}\right)-\gamma u_{1} u_{2} \\
& \frac{\partial u_{2}}{\partial t}=-\gamma u_{1} u_{2} .
\end{aligned}
$$

Diffusion and reaction terms are not commuted here since the reaction term is non-linear. Thus, a splitting error of first order $o(\tau)$ is expected to occur. 


\subsection{The Finite Element Method}

In this section, we present the finite element to solve numerically the system of partial differential equations (5) and (6) together with the Dirichlet boundary conditions $u_{1}(0, t)=u_{1}(L, t)=1$ and $u_{2}(0, t)=$ $u_{2}(L, t)=0$ for $t>0$ and dividing the computational domain $0 \leq x \leq L$.

The weak formulation for the problem (5) and (6) read as follows: For every time interval $I_{n}=$ $\left(t_{n-1}, t_{n}\right]$ find $u(x, t), t \in I_{n}$ such that

$$
\begin{gathered}
\int_{t_{n-1}}^{t_{n}} \int_{0}^{L} v(x)\left(\frac{\partial u_{1}}{\partial t}-D_{1} \frac{\partial^{2} u_{1}}{\partial x^{2}}\right) d x d t=0 \\
\int_{t_{n-1}}^{t_{n}} \int_{0}^{L} v(x)\left(\frac{\partial u_{2}}{\partial t}-D_{2} \frac{\partial^{2} u_{2}}{\partial x^{2}}\right) d x d t=0
\end{gathered}
$$

where $v(x)$ is arbitrary test function. On applying integration by part with the conditions on the second order derivative in (9) and (10) and after simplification it reduces to the form

$\int_{t_{n-1}}^{t_{n}}\left[\int_{0}^{L}\left(\frac{\partial u_{1}}{\partial t} v+D_{1} \frac{\partial u_{1}}{\partial x} \frac{\partial v}{\partial x}\right) d x\right] d t=0, \forall v: v(0, t)=v(L, t)=0$.

$\int_{t_{n-1}}^{t_{n}}\left[\int_{0}^{L}\left(\frac{\partial u_{2}}{\partial t} v+D_{2} \frac{\partial u_{2}}{\partial x} \frac{\partial v}{\partial x}\right) d x\right] d t=0, \forall v: v(0, t)=v(L, t)=0$

A piecewise linear Galerkin approximation: For each time interval $I_{n}=\left(t_{n-1}, t_{n}\right]$ with step size $k=$ $t^{n}-t^{n}-1$, let

$u(x, t)=u_{n-1}(x) \varphi_{n-1}(t)+u_{n}(x) \varphi_{n}(t)(13)$

Here in (13), we used the notations $\varphi_{n}(t)=\frac{t-t_{n}}{k}, \varphi_{n-1}(t)=\frac{t_{n-1}-t}{k}$ and

$u_{n}(x)=u_{n, 1} \varphi_{1}(x)+u_{n, 2} \varphi_{2}(x)+\ldots+u_{n, m} \varphi_{m}(x)$. (14)

In (13), $u$ is piecewise linear function with respect to both space and time variables.

By the Galerkin approach, we choose the test function to be the same as the basis function. Hence the unknowns are the coefficients $u_{n, k}$ satisfying discrete weak formulation as

$$
\begin{aligned}
\int_{t_{n-1}}^{t_{n}}\left[\int_{0}^{L}\left(\frac{\partial u_{1}}{\partial t} \varphi_{j}+D_{1} \frac{\partial u_{1}}{\partial x} \frac{\partial \varphi_{j}}{\partial x}\right) d x\right] d t=0 \\
\int_{t_{n-1}}^{t_{n}}\left[\int_{0}^{L}\left(\frac{\partial u_{2}}{\partial t} \varphi_{j}+D_{2} \frac{\partial u_{2}}{\partial x} \frac{\partial \varphi_{j}}{\partial x}\right) d x\right] d t=0
\end{aligned}
$$

Here $j$ refers to the number of nodes in an element and $j=1,2, \ldots, m$.

Further, on substituting the step size $k=t_{n}-t_{n-1}$ and the space variables $u_{n}=u\left(x_{n}\right)$ and $u_{n-1}=$ $u\left(x_{n-1}\right)$ the equation (13) takes the form as

$\dot{u}(x, t)=u_{n-1}(x) \varphi_{n-1}{ }^{\prime}(t)+u_{n}(x) \varphi_{n}{ }^{\prime}(t)=\frac{u_{n}-u_{n-1}}{k}(17)$

Also differentiation of (13) with respect to $x$ gives

$u^{\prime}(x, t)=u_{n-1}^{\prime}(x) \varphi_{n-1}(t)+u_{n}^{\prime}(x) \varphi_{n}(t)(18)$

Up on inserting (17) and (18) and using the values of definite integrals $\int_{t_{n-1}}^{t_{n}} d t=k$ and $\int_{t_{n-1}}^{t_{n}} \varphi_{n} d t=$ $\int_{t_{n-1}}^{t_{n}} \varphi_{n-1} d t=\frac{k}{2}$ the equations (15) and (16) take the form as

$\int_{0}^{L} u_{1 n} \varphi_{j} d x-\int_{0}^{L} u_{1(n-1)} \varphi_{j} d x+D_{1} \frac{k}{2} \int_{0}^{L} u_{1(n-1)}^{\prime} \varphi_{j}^{\prime} d x+D_{1} \frac{k}{2} \int_{0}^{L} u_{1 n}^{\prime} \varphi_{j}^{\prime} d x=0$ (19)

$\int_{0}^{L} u_{2 n} \varphi_{j} d x-\int_{0}^{L} u_{2(n-1)} \varphi_{j} d x+D_{2} \frac{k}{2} \int_{0}^{L} u_{2(n-1)}^{\prime} \varphi_{j}^{\prime} d x+D_{2} \frac{k}{2} \int_{0}^{L} u_{2 n}^{\prime} \varphi_{j}^{\prime} d x=0$ (20)

These equations can be written in the compact form as Crank-Nicolson system (CNS)

$$
\begin{aligned}
& u_{1 n}=\left(M+\frac{k}{2}\left(D_{1} A_{d}\right)\right)^{-1}\left(M-\frac{k}{2}\left(D_{1} A_{d}\right)\right) u_{1(n-1)} \\
& u_{2 n}=\left(M+\frac{k}{2}\left(D_{2} A_{d}\right)\right)^{-1}\left(M-\frac{k}{2}\left(D_{2} A_{d}\right)\right) u_{2(n-1)}
\end{aligned}
$$

To compute the stiffness matrices of diffusion term $A_{d}$ and the mass matrix $M$, let us differentiate (14) to obtain $u_{n}^{\prime}(x)=u_{n, 1} \varphi_{1}^{\prime}(x)+u_{n, 2} \varphi_{2}^{\prime}(x)+\ldots+u_{n, m} \varphi_{m}^{\prime}(x)$. Thus, we have

$$
A_{d} u_{n}=\int_{0}^{L} u_{n}^{\prime} \varphi_{j}^{\prime} d x=\left(\int_{0}^{L} \varphi_{j}^{\prime} \varphi_{1}^{\prime}\right) u_{n, 1} d x+\left(\int_{0}^{L} \varphi_{j}^{\prime} \varphi_{2}^{\prime}\right) u_{n, 2} d x+\ldots+\left(\int_{0}^{L} \varphi_{j}^{\prime} \varphi_{m}^{\prime}\right) u_{n, m} d x \text {. }
$$

By using the linear piecewise function $\varphi_{j}(x)$ with uniform mesh

$$
\varphi_{j}(x)=\left\{\begin{array}{cc}
\frac{x-x_{j-1}}{h}, & x \in\left[x_{j-1}, x_{j}\right] \\
\frac{x_{j+1}-x}{h}, & x \in\left[x_{j}, x_{j+1}\right]^{(23)} \\
0, & \text { else. }
\end{array}\right.
$$

Then, we obtain $A_{d}=\frac{1}{h} \operatorname{tridiag}[-1,2,-1] \in R^{(m-1) \times(m-1)}$. 
Similarly, for all the indices $j=1,2, \ldots, m$ the mass matrix $M$ is defined as

$$
M u_{n}=\int_{0}^{L} u_{n} \varphi_{j} d x
$$

With the function (23) for uniform partition, we obtain $M=\frac{h}{6} \operatorname{tridiag}[1,4,1] \in R^{(m-1) \mathrm{X}(m-1)}$.

\subsection{Improved Runge-Kutta Method of Order Six}

Here we adopt the improved Runge - Kutta method of order six in the form that was used in our earlier study [20]. However, for the ready reference the algorithm is presented here once again. The algorithm is given as

$u_{6}\left(t_{0}+\mathrm{h}\right)=u_{0}+\frac{1}{840}\left[41\left(k_{0}+k_{7}\right)+216\left(k_{2}+k_{6}\right)+27\left(k_{3}+k_{5}\right)+272 k_{4}\right]$

Here in (24), the quantities $k_{i}, \forall i=0,1, \ldots, 7$ represent

$k_{0}=\mathrm{h} f\left(t_{0}, u_{0}\right)$

$k_{1}=\mathrm{h} f\left(t_{0}+\frac{1}{9} \mathrm{~h}, u_{0}+\frac{1}{9} k_{0}\right)$

$$
\begin{gathered}
k_{2}=\mathrm{h} f\left(t_{0}+\frac{1}{6} \mathrm{~h}, u_{0}+\frac{1}{24}\left(k_{0}+3 k_{1}\right)\right) \\
k_{3}=\mathrm{h} f\left(t_{0}+\frac{1}{3} h, u_{0}+\frac{1}{6}\left(k_{0}-3 k_{1}+4 k_{2}\right)\right) \\
k_{4}=\mathrm{h} f\left(t_{0}+\frac{1}{2} \mathrm{~h}, u_{0}+\frac{1}{8}\left(k_{0}+3 k_{3}\right)\right)
\end{gathered}
$$

$k_{5}=\mathrm{h} f\left(t_{0}+\frac{2}{3} \mathrm{~h}, u_{0}+\frac{1}{9}\left(17 k_{0}-63 k_{1}+51 k_{2}+k_{4}\right)\right)$

$k_{6}=\mathrm{h} f\left(t_{0}+\frac{5}{6} \mathrm{~h}, u_{0}+\frac{1}{24}\left(-22 k_{0}+33 k_{1}+30 k_{2}-58 k_{3}+34 k_{4}+3 k_{5}\right)\right)$

$k_{7}=\mathrm{h} f\left(t_{0}+\mathrm{h}, u_{0}+\frac{1}{82}\left(281 k_{0}-243 k_{1}-522 k_{2}+876 k_{3}-346 k_{4}-36 k_{5}+72 k_{6}\right)\right)$.

The reaction term given in $(7)-(8)$ is solved using improved sixth order formula together with initial conditions of $u_{1}$ and $u_{2}$ and with the inclusion of parametric values.

\section{Numerical Results}

Let us now compute numerical solutions for the system of equations (3) and (4) by restricting the independent variables to the regions $0 \leq x \leq 1$ and $t>0$. The initial conditions are considered as $u_{1}(x, 0)=$ $1 \mathrm{kgm}^{-3}$ and $u_{2}(x, 0)=6 \mathrm{kgm}^{-3}$. Similarly, the boundary conditions are chosen as $u_{1}(0, t)=u_{1}(1, t)=$ $1 \mathrm{kgm}^{-3}$ and $u_{2}(0, t)=u_{2}(1, t)=0 \mathrm{kgm}^{-3}$. Also, the parametric values are set as $\omega=1 \mathrm{kgm}^{-3}$ and $\gamma=$ $1 \mathrm{day}^{-1}$. Then, we vary the parameters $D_{1}, D_{2}$ and $\lambda$ to study the effects of reaction, diffusion and reaeration in river pollution.

\subsection{Zero Diffusion}

Consider the case when diffusion taken to be zero, i.e. $D_{1}=D_{2}=0$. In this case the system of diffusion-reaction equations (3) and (4) reduces to a system of reaction equations as given in (7) and (8). This resembles to a well - mixed case. Thus, we are only studying the effect of self-purification, without considering the spatial distribution. We now employ the method of improved Runge-Kutta of order six on the system of equations given in (7) and (8). For this purpose we consider the initial conditions and parametric values given in Section 4. The results of the simulation study are given in Figures 1 and 2.

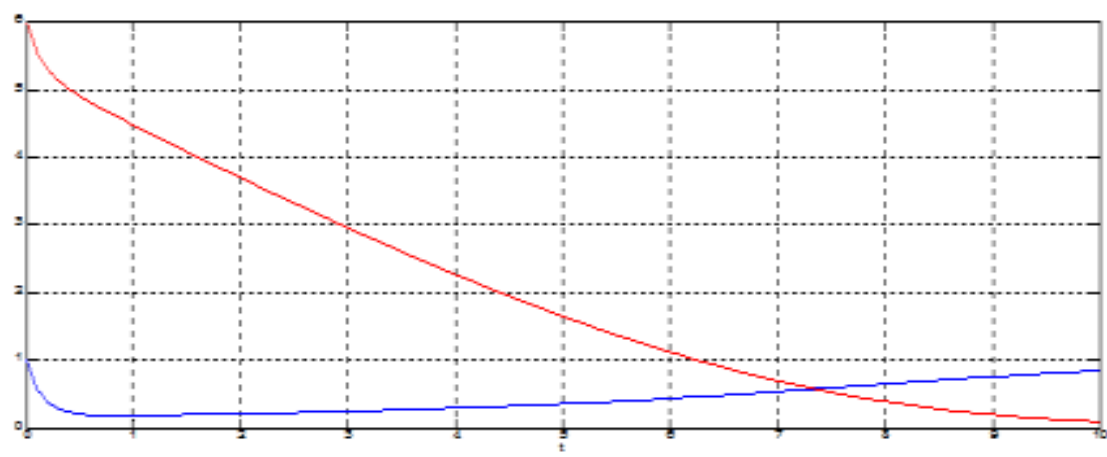


Figure 1: Numerical simulation of the system of equations (7) and (8) with zero diffusion term and $\lambda=1 m^{2} d a y^{-1}$.

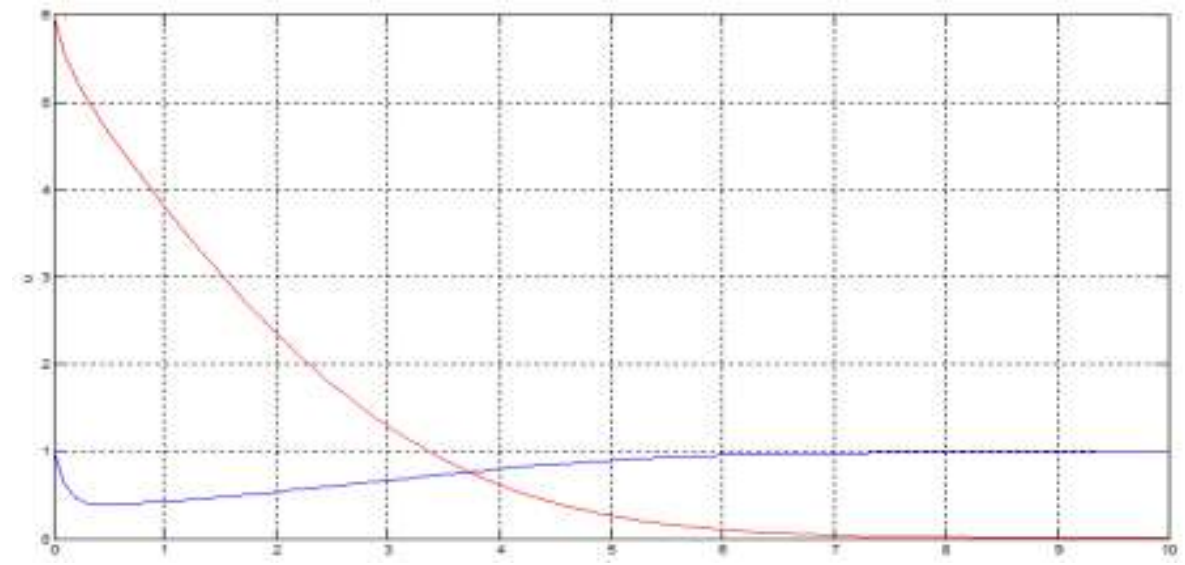

Figure 2: Numerical simulation of the system of equations (7) and (8) with zero diffusion term and

$$
\lambda=3 m^{2} d a y^{-1} \text {. }
$$

In Figures 1 and 2, the blue and red curves represent the amounts of dissolved oxygen (DO), $u_{1}$ and the biochemical oxygen demand (BOD), $u_{2}$ respectively at a given time in the river. At time $t=0$, the amount of DO is $u_{1}(t)=1 \mathrm{kgm}^{-3}$ and the amount of BOD is $u_{2}(t)=6 \mathrm{kgm}^{-3}$. Since the BOD consumes DO and as a result both the BOD and DO availability are declined. Now in this situation the self-purification capacity of the river becomes active. This helps $u_{1}$ in performing two activities: (i) to reduce $u_{2}$ to zero and (ii) to increase $u_{1}$ to reach its normal value 1 due to the effect of the re-aeration or the term $\lambda\left(\omega-u_{1}\right)$. This process is slower with smaller re-aeration effects as seen in Figure 1 and faster with larger re-aeration effects as seen in Figure 2.

In other words, Figures 1 and 2 can be interpreted as follows: At $t=0$, biological waste material or pollutant is added to the river water with BOD concentration 6 times as high as DO concentration. The biological waste material immediately reacts with the DO causing the concentration to drop. Also the BOD concentration caused to drop. At a later time, the self-cleaning system of the water takes a leading role, so BOD concentration goes down to zero and the DO concentration goes up its normal value 1 due to re-aeration effects. For a high re-aeration rate $\lambda$, the DO concentration immediately goes to its normal value and BOD concentration goes down to zero rapidly as figure 2 describes.

\subsection{Including Diffusion}

We now consider that both the diffusion coefficients $D_{1}$ and $D_{2}$ appear in the system of equation (3) and (4) are different from zero, i.e., $D_{1} \neq 0$ and $D_{2} \neq 0$. Hence, the equations remain to be a system of diffusionreaction equations. Note that splitting the diffusion term from reaction term has computational advantages since simultaneous coupling over space and the various chemical species is then avoided, and it also offers room for massively parallel computing. Thus, using the discretization of diffusion term given in equations (21) and (22) and algorithms of an improved Runge-Kutta of order six given in [20], we have the simulation results with different diffusion and reaeration coefficients as shown in Figures 3 - 6.
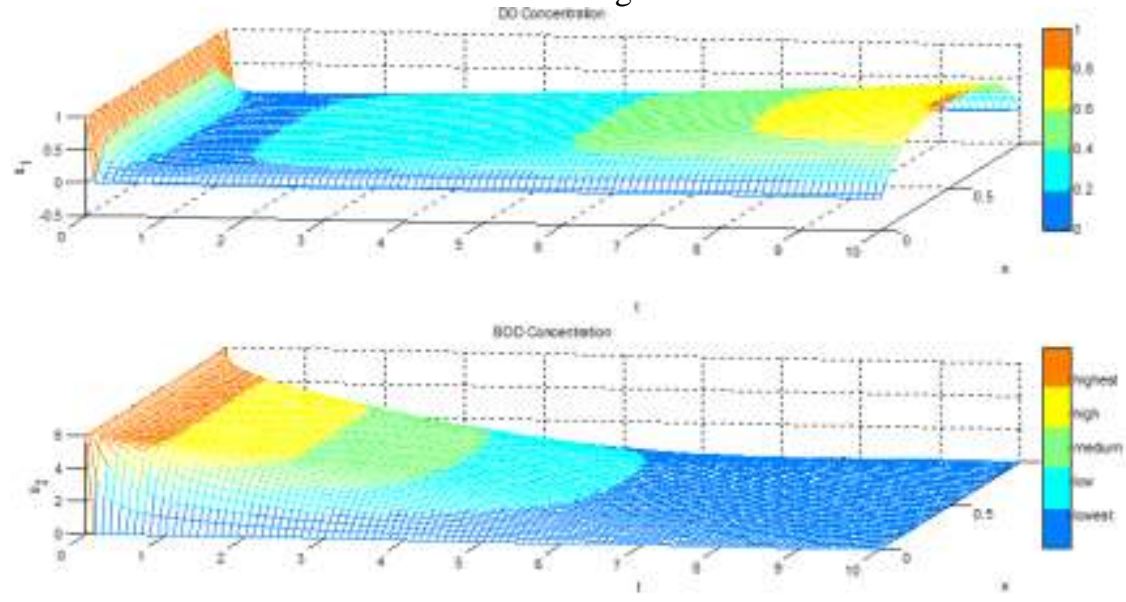
Figure 3: Numerical solution for including diffusion with $D_{1}=0.01 \mathrm{~m}^{2} \mathrm{day}^{-1}, D_{2}=0.001 \mathrm{~m}^{2} \mathrm{day}^{-1}$ and $\lambda=1 m^{2}$ day $^{-1}$.
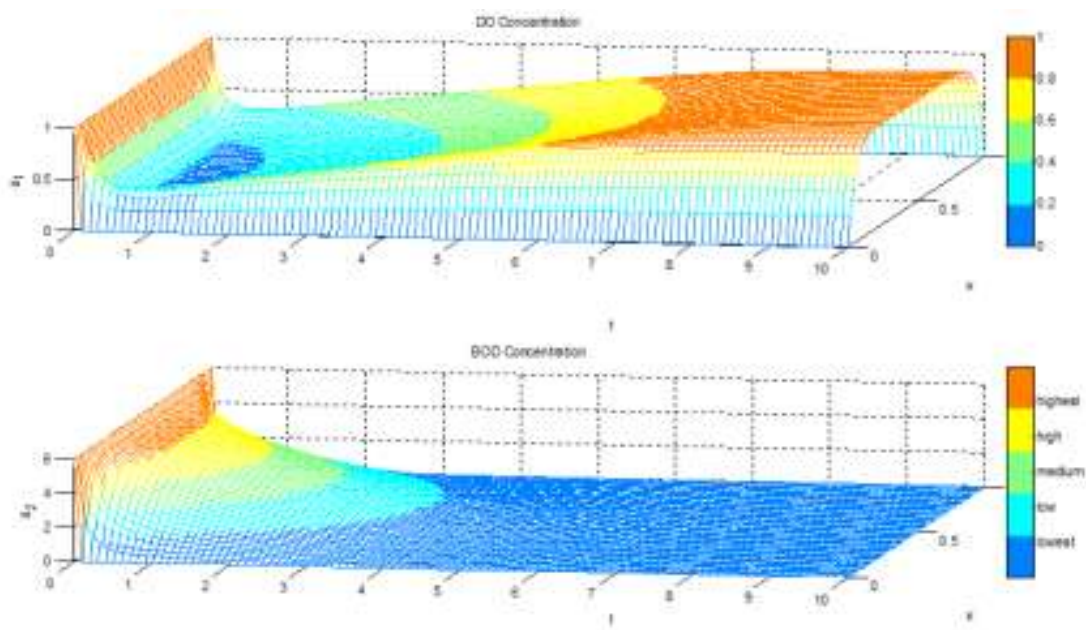

Figure 4: Numerical solution for including diffusion with $D_{1}=0.001 \mathrm{~m}^{2} d a y^{-1}, D_{2}=0.01 \mathrm{~m}^{2} \mathrm{day}^{-1}$ and $\lambda=1 m^{2}$ day $^{-1}$.
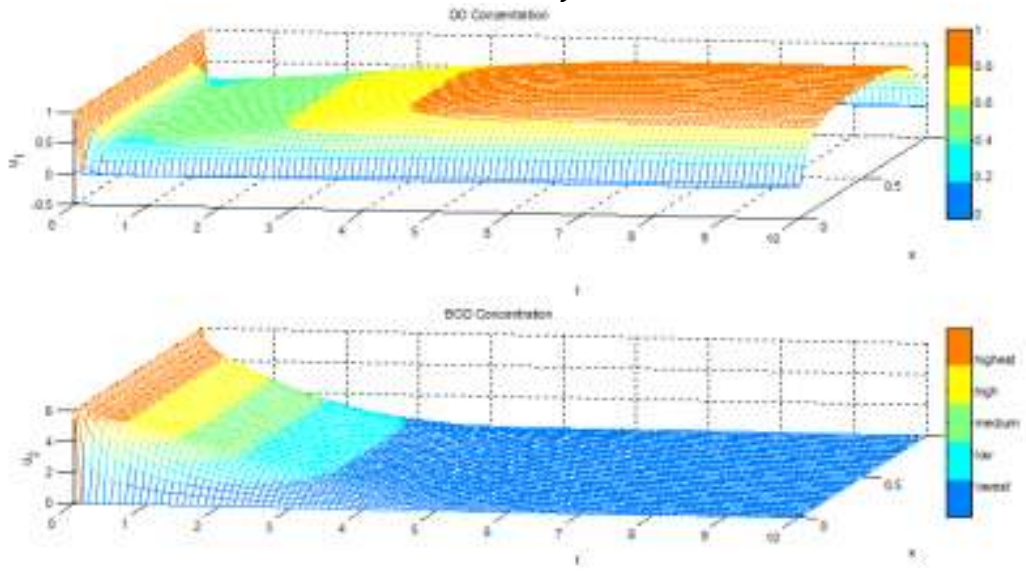

Figure 5: Numerical solution for including diffusion with $D_{1}=0.01 \mathrm{~m}^{2} \mathrm{day}^{-1}, D_{2}=0.001 \mathrm{~m}^{2} \mathrm{day}^{-1}$ and

$$
\lambda=3 m^{2} d a y^{-1} \text {. }
$$
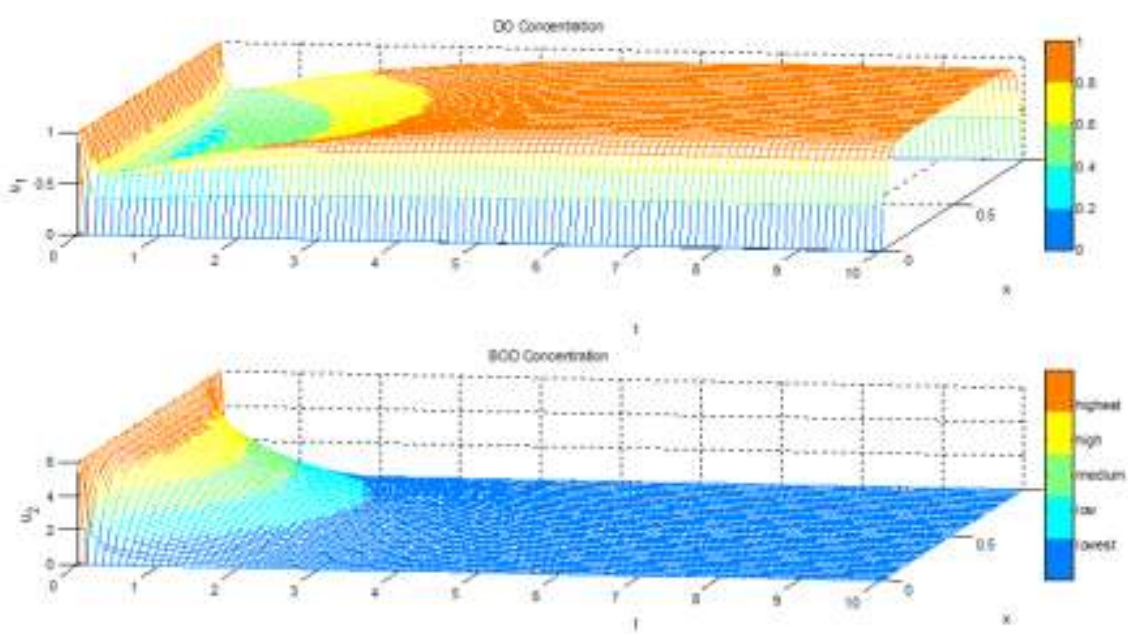

Figure 6: Numerical solution for including diffusion with $D_{1}=0.001 \mathrm{~m}^{2} \mathrm{day}^{-1}, D_{2}=0.01 \mathrm{~m}^{2} \mathrm{day}^{-1}$ and

$$
\lambda=3 m^{2} d a y^{-1} \text {. }
$$


In the Figures $3-6$, we ignore all variations along the flow direction and depth of the river, but only observe along the cross-sectional area of a river of width one unit. At timet $=0$, waste water is poured into the river water all over. But we assume that the water at the boundaries is clean and so there, the oxygen concentration is 1 , and the BOD concentration is zero. But then, in addition to the self-cleaning effect from the reactive term, we get diffusion of the clean water from the boundaries which make the river clean faster than if we did not have this effect. As shown in figures, whenever the parameters $D_{1}, D_{2}$ and $\lambda$ assume larger values the river gets cleaned faster. However, the same statement can be equivalently stated as: the parameters $D_{1}, D_{2}$ and $\lambda$ assume smaller values the river gets cleaned slower.

\section{Conclusions}

In this study, we have presented one-dimensional and unsteady coupled pair of nonlinear diffusionreaction equations to describe the environmental purification model and also computed its solutions using different numerical methods. We applied the results to two test cases and verified. Of these,the first being a zero diffusion case, while the other is a non-zero diffusion case. In the former case we have observed that the selfpurification of river takes place and the purification process is slower. However, in the latter case because of the diffusion coefficients of DO and BOD, the purification process of the river becomes so fast. In both cases with higher reaeration coefficient the river cleans faster. The algorithm has been implemented in Matlab, which was also used in generating all the graphics presented in the text.

\section{References}

[1]. Brain J. McCartin, Sydney B. and Forrester Jr. (2002), A Fractional Step-Exponentially Fitted Hopscotch Scheme for the StreeterPhelps Equations of River Self-Purification, Engineering Computations, Vol. 19, Pp. 177-189.

[2]. Butcher J. C. (2009), On Fifth and Sixth Order Explicit Runge-Kutta Methods: Order Conditions and Order Barriers, Canadian Applied Mathematics Quarterly, Vol. 17, No. 3, Pp.1-14.

[3]. Chertock A., Kurganov A.and Petrova G. (2006), Fast explicit operator splitting method for convection-diffusion equations, International Journal for Numerical Methods in Fluids, doi: 10.1002/fld.1355, http://www.interscience.wiley.com.

[4]. Geiser J. (2016), MulticomponentandMultiscaleSystems Theory, Methods and Applications in Engineering, Springer International Publishing Switzerland, doi 10.1007/978-3-319-15117-5_2, http://www.springer.com/978-3-319-15116-8.

[5]. HasadsriS. and MaleewongM. (2012), Finite Element Method for Dissolved Oxygen and Biochemical Oxygen Demand in an Open Channel, Procedia Environmental Sciences, Vol. 13, Pp. 1019 - 1029, doi:10.1016/j.proenv.2012.01.095.

[6]. Holden H., Karlsen K.H., Lie K. and Risebro N.H, (2010), Splitting Methods for Partial Differential Equations with Rough Solutions-Analysis and MATLAB programs, European Mathematical Society, ISBN 978-3-03719-078-4.

[7]. Hundsdorfer W. and Verwer J.G. (2003), Numerical Solution of Time Dependent Advection-Diffusion-Reaction Equations, Springer-Verlag, Berlin Heidelberg.

[8]. Hussain S.A., Atshan W.G. and Najam Z.M. (2012), Mathematical Model for the Concentration of Pollution and Dissolved Oxygen in the Diwaniya River (Iraq),American Journal of Scientific Research, ISSN 2301-2005 Issue 78, Pp.33-37, http://www.eurojournals.com/ajsr.htm.

[9]. Kaushik N., Tyagi B. and Jayaraman G. (2012), Modeling of the Dissolved Oxygen in a River with Storage Zone on the Banks, Applied Mathematics, Vol. 3, Pp. 699-704, http://dx.doi.org/10.4236/am.2012.37103.

[10]. Mahto K. and Kumar I. (2015), The Self Purification Model for Water Pollution,International Journal of Mathematics and Statistics Invention (IJMSI), Vol. 3, Pp. 17-32, www.Ijmsi.org.

[11]. Mesteron-Gibbons, M. (2007), A Concrete Approach to Mathematical Modeling, John Wiley and Sons - Interscience.

[12]. Pimpunchat B., Sweatman W.L., Wake G.C., Triampo W. and Parshotam A. (2009), A Mathematical Model for Pollution in River and Its Remediation by Aeration, Applied Mathematics Letters, Vol. 22, Pp. 304-8, doi:10.1016/j.aml.2008.03.026.

[13]. Pimpunchat B., Sweatman W.L., Wake G.C., Triampo W. and Parshotam A., Modeling River Pollution and Removal by Aeration.

[14]. Quarteroni A. (2009), Numerical Models for Differential Problems, $4^{\text {th }}$ ed., Springer-Verlag Italia.

[15]. Ramalinga Reddy D.V and Sreenivasa Chari A. (2013), A Mathematical Model for Toxic Waste in a River and Its Remediation by Freshening, International Journal of Innovative Research \& Studies, Vol. 2, Pp. 1-10.

[16]. Sanderson A.R., Meyer M.D., Kirby R.M. and Johnson C.R. (2007), A Frame works for Exploring Numerical Solutions of Advection-Reaction-Diffusion Equations Using a GPU Based Approach, Computer visual Sci., Vol. 10, Pp. 1-16.

[17]. Sarafyan D. (1972), Improved Sixth-Order Runge-Kutta Formulas and Approximate Continuous Solution of Ordinary Differential Equations, Journal of Mathematical Analysis and Applications, Vol. 40, Pp. 436-445.

[18]. Shukla J.B., Misra A.K. and Chandra P. (2008), Mathematical Modeling and Analysis of the Depletion of Dissolved Oxygen in Eutrophied Water Bodies Affected by Organic Pollutants, Nonlinear Analysis: Real World Applications, Vol. 9, Pp. 1851-1865, doi:10.1016/j.nonrwa.2007.05.016.

[19]. Simon T. and Koya P.R. (2015), Modeling and Numerical Simulation of River Pollution Using Diffusion-Reaction Equation, American Journal of Applied Mathematics, Vol. 3, No. 6, Pp. 335-340, doi: 10.11648/j.ajam.20150306.24.

[20]. Simon T. and Koya P.R. (2016), Numerical Simulation of System of Time-Dependent Advection-Diffusion-Reaction Equations Describing River Pollution, International Journal of Scientific Progress and Research, Vol. 30, Pp. 94-106.

[21]. Sportisse B. (2007), A Review of Current Issues in Air Pollution Modeling and Simulation, ComputGeosci, Vol. 11, PP.159-181.

[22]. Streeter H.W. and Phelps E.B. (1925), A Study of the Pollution and Natural Purification of the Ohio River, U.S. Public Health Service, Bulletin No. 146.

[23]. Verwer J., Hundsdorfer G., Willem H. and Joke G. (2002), Numerical Time Integration for Air Pollution Models, Surv. Math. Ind., Vol. 10, Pp.107-174.

[24]. Zingg D.W. and Chisholm T.T. (1999), Runge-Kutta methods for linear ordinary differential equations, Applied Numerical Mathematics, Vol. 31, Pp. 227-238. 